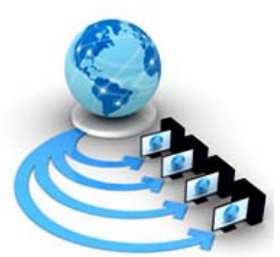

\title{
CONTENT BASED IMAGE RETRIEVAL SYSTEM USING SHAPE TEXTURE AND COLOR FEATURES
}

\author{
Parul Nangia \\ Research Scholar,Department of Computer Science \\ Shaheed Bhagat Singh State Technical Campus \\ Ferozepur, Punjab
}

\author{
Sonika Jindal \\ Assistant Professor, Department of Computer Science \\ Shaheed Bhagat Singh State Technical Campus \\ Ferozepur, Punjab
}

\begin{abstract}
Content based image retrieval is an important field of multimedia applications. This has been used for extraction of similar images from the large datasets. Systems that have been used for content based image retrieval system use various features that are low level. Various approaches and features have been widely used for extraction of relevant information. In this paper a new approach has been proposed that use combination of colours, shape and texture features. SURF and Fuzzy has been combined in this system for efficient retrieval of the relevant images. SURF is a fast approach that compute point based descriptions about the shape features of the images. Fuzzy interference has been used for computation of fuzzy membership values so that efficient feature vector can be developed. On the basis of parameters analysis we can predict that proposed approach provide better performance.
\end{abstract}

Keywords: CBIR, SURF, Fuzzy and GLCM

\section{INTRODUCTION}

IMAGE indexing and retrieval is demanding more and more attention due to its rapid growth in many places. Image retrieval has several applications such as in object recognition, biomedical, agriculture, etc. The aim of Content Based Image Retrieval (CBIR) [1] is to extract the similar images of a given image from huge databases by matching a given query image with the images of the database. Matching of two images is facilitated by the matching of actually its feature descriptors. It means the performance of any image retrieval system heavily depends upon the image feature descriptors being matched. Color, texture, shape, gradient, etc. are the basic type of features to describe the image. Texture based image feature description is very common in the research community.

\subsection{Content-based image retrieval (CBIR)}

Content-based image retrieval (CBIR), also known as query by image content (QBIC) [2] and content-based visual information retrieval (CBVIR) is the application of machine vision strategies to the picture recovery issue, that is, the issue of hunting down computerized pictures in huge databases .Substance based picture recovery is restricted to customary idea based methodologies. CBIR is attractive in light of the fact that ventures that depend simply on metadata are reliant on annotation quality and culmination. Having peopled physically clarifies pictures by entering decisive words or metadata in an extensive database can be drawn out and may not catch the catchphrases wanted to depict the picture. The assessment of the viability of catchphrase picture hunt is subjective and has not been decently characterized. In the same respect, CBIR frameworks have comparative difficulties in characterizing achievement.

\subsection{CBIR Techniques}

1.2.1 Query Techniques: Diverse executions of CBIR make utilization of distinctive sorts of client questions. Question by illustration is an inquiry procedure that includes furnishing the CBIR framework with a case picture that it will then base its pursuit upon. The hidden inquiry calculations may fluctuate relying upon the application; however result images should all share normal components with the given sample.

1.2.2 Semantic retrieval: Semantic recovery [3] begins with a client making an appeal like "discover pictures of Abraham Lincoln”. This sort of open-finished undertaking is extremely troublesome for machines to perform - Lincoln may not generally be confronting the cam or in the same stance. Numerous CBIR frameworks accordingly for the most part make utilization of lower-level gimmicks like composition, color, and shape. These gimmicks are either utilized as a part of mix with interfaces that permit simpler data of the criteria or with databases that have as of now been prepared to match peculiarities.

1.2.3 Relevance Feedback: Consolidating CBIR [3] seeks procedures accessible with the extensive variety of potential clients and their goal can be a troublesome errand. A part of making CBIR fruitful depends completely on the capacity to comprehend the client intent. CBIR frameworks can make utilization of pertinence input, where the client dynamically refines the query items by stamping pictures in the results as "important", "not "significant", or "nonpartisan" to the inquiry question, then rehashing the hunt with the new data. Illustrations of this kind of interface have been developed.

1.2.4 Iterative/Machine Learning: Machine learning and application of iterative systems are getting to be more basic in CBIR.

1.2.5 Other query methods: Other inquiry strategies incorporate searching for instance pictures, exploring tweaked/various leveled classes, questioning by picture district (instead of the whole picture), questioning by numerous illustration pictures, questioning by visual portrayal, questioning by immediate detail of picture gimmicks, and multimodal inquiries.

1.3 CBIR techniques: In contrast to the text-based approach of the systems described, CBIR operates on a totally different principle, retrieving stored images from a collection by 
comparing features automatically extracted from the images themselves. The commonest features used are mathematical measures of color, texture or shape hence virtually all current CBIR systems, whether commercial or experimental, operate at level 1. A typical system allows users to formulate queries by submitting an example of the type of image being sought, though some offer alternatives such as selection from a palette or sketch input. The system then identifies those stored images whose feature values match those of the query most closely, and displays thumbnails of these images on the screen. Color retrieval [5] several methods for retrieving images on the basis of color similarity have been described in the literature, but most are variations on the same basic idea. Each image added to the collection is analyzed to compute a color histogram which shows the proportion of pixels of each color within the image. The color histogram for each image is then stored in the database.

1.3.1 Texture retrieval: The ability to retrieve images on the basis of texture similarity [4] may not seem very useful. But the ability to match on texture similarity can often be useful in distinguishing between areas of images with similar color. A variety of techniques has been used for measuring texture similarity; the best-established rely on comparing values of what are known as second-order statistics calculated from query and stored images. Essentially, these calculate the relative brightness of selected pairs of pixels from each image. From these it is possible to calculate measures of image texture such as the degree of contrast, coarseness, directionality and regularity or periodicity, directionality and randomness. Alternative methods of texture analysis for retrieval include the use of Gabor filters and fractals. Texture queries can be formulated in a similar manner to color queries, by selecting examples of desired textures from a palette, or by supplying an example query image.

1.3.2 Shape retrieval: The ability to retrieve by shape is perhaps the most obvious requirement at the primitive level. Unlike texture, shape is a fairly well-defined concept -and there is considerable evidence that natural objects are primarily recognized by their shape [6]. A number of features characteristic of object shape are computed for every object identified within each stored image. Queries are then answered by computing the same set of features for the query image, and retrieving those stored images whose features most closely match those of the query. Two main types of shape feature are commonly used -global features such as aspect ratio, circularity and moment invariants and local features such as sets of consecutive boundary segments.

\subsection{Applications of CBIR}

- Crime prevention: Automatic face recognition systems, used by police forces.

- Security Check: Finger print or retina scanning for access privileges.

- Medical Diagnosis: Using CBIR in a medical database of medical images to aid diagnosis by identifying similar past cases.

- Intellectual Property: Trademark image registration, where a new candidate mark is compared with existing marks to ensure no risk of confusing property ownership.

\section{REVIEW OF LITERATURE}

Shiv Ram Dubey et al [7] "Multichannel Decoded Local Binary Patterns for Content-Based Image Retrieval” Image retrieval experiments are performed to observe the effectiveness of the proposed approaches and compared with the existing ways of multichannel techniques. The experiments are performed over 12 benchmark natural scene and color texture image databases, such as Corel-1k, MIT-VisTex, USPTex, Colored Brodatz, and so on. It is observed that the introduced multichannel adder- and decoder-based LBPs significantly improve the retrieval performance over each database and outperform the other multichannel-based approaches in terms of the average retrieval precision and average retrieval rate.

C. R. Shyuet al [8] "Assert: A physician-in-the-loop contentbased retrieval system for hrct image databases.” This system was developed at Purdue University. It was designed especially for high resolution computed tomography images, since it uses some perceptual features specific to those images. It also include gray-level features, like mean and standard deviation, texture features like contrast, entropy and homogeneity and shape description such as the area. The feature vectors are indexed using the multi-hash method.

T. M. Lehmann et al [8] "IRMA - a content-based approach to image retrieval in medical applications,” The IRMA system was developed on the Aachen University of Technology. It is focused on the querying of medical images using manually defined prototypes in a first stage and in features extracted from frequency, texture and structure analysis in regions segmented in multi-scale blob-representation. Those features are then indexed using a cluster-based approach.

W. Y. Ma et al [10] "Netra: A toolbox for navigating large image databases,” Nitra image retrieval system was developed at UC Santa Barbara. This system utilizes Color, shape, spatial location and texture information of segmented image to explore and retrieve alike regions from database. It enables the users to frame queries like, retrieve each and every image which include regions that have similar color, texture and shape of objects, and lie in the upper one-third of image, where individual objects region can belongs to dissimilar images.

N. Boujemaaet al [11] "IKONA: Interactive generic and specific image retrieval," Ikona is prototype application designed for the IMEDIA, and developed at INRIA. Ikona system makes use of query-by-example approach for retrieving similar images and also integrates superior attributes like image signature and face recognition. It supplies hybrid text-image retrieval method and query modification with relevance feedback, mutually with a region-based approach in which the user is capable to choose an element from image and system searches images which are visually analogous to selected element.

J. Z. Wang, et al [12] "SIMPLICITY: Semantics-sensitive integrated matching for picture libraries," Semantics-sensitive Integrated Matching for Picture Libraries image retrieval system developed by Stanford University, in which images are represented via a set of regions that nearly corresponds to items that can be categorized via their color, shape, location and texture properties. Segmentation of region is achieved by a straightforward algorithm based on k-means clustering in 
feature space. As opposed to region-wise retrieval, images are retrieved as a whole with the help of region matching techniques which incorporate properties of regions within the image to measure similarity. This overall similarity approach is to decrease the influence of inaccurate segmentation.

\section{METHDOLOGY}

Content based image retrieval is the process of extraction of relevant images from the dataset based on features. In this paper a novel approach has been prescribed that has been used for extraction of relevant information based on shape, texture and colors available in the query images. In this process of feature extraction a combination of different approaches have been utilized that computes various features from the images and store in a single feature vector.

In the process of content based image retrieval system features from query image has been extracted and utilized for matching process based on distance classifier.

In proposed model a color based features have been extorted using fuzzy based color histogram approach. This approach has been used for extraction of color features from the images. On the basis of color spaces images have been converted into different color spaces o that color information can be extracted. This information has been provided as input to fuzzy interference model. A set of fuzzy rules has been used for generation of fuzzy membership values. These values have been used as feature vector for color descriptions.

SURF that is a (Speeded up Robust Feature) is an approach that has been used for extraction of shape based features from the images. This approach is fast approach that is scale and rotation invariant. This approach computes features very fast so processing of the algorithm decreases time complexity of overall system. It uses Integral images to improve the speed. The key points are detected by using a Fast-Hessian matrix. The descriptor describes a distribution of Haar-wavelet responses within the interest point neighborhood. The performance of SURF has been increased by using an intermediate image representation known as the Integral Image. The integral image is computed rapidly from an input image and is used to speed up the calculation of any upright rectangular area.

- The SURF feature detector is based on the Hessian matrix. The determinant of the Hessian matrix is used to determine the location and scale of the descriptor. In order to localize interest points in the image and over scales, non-maximum suppression in a $3 \times 3 \times 3$ neighborhood is applied. Finally, the found maxima of the determinant of the Hessian matrix are then interpolated in scale and image space.

- In a second step, the region is split up regularly into smaller square sub-regions and a few simple features at regularly spaced sample points are computed for each sub-region. The horizontal and vertical wavelet responses are summed up over each sub-region to form a first set of entries to the feature vector. The responses of the Haar-wavelets are weighted with a Gaussian centered at the interest point in order to increase robustness to geometric deformations and the wavelet responses in horizontal $\mathrm{dx}$ and vertical Directions dy are summed up over each sub-region.
Furthermore, the absolute values $|\mathrm{dx}|$ and $|\mathrm{dy}|$ are summed in order to obtain information about the polarity of the image intensity changes.

Texture features have been computed from the images using Gray level co-occurrence matrix. GLCM has been used for generation of 8 by 8 matrixes from the input image that computes value of the texture features. These features are contrast, energy homogeneity and correlation has been measured. These have been computed using various mathematical expressions that are listed below.

$$
\begin{aligned}
& \text { Contrast }=\sum_{\mathrm{i}_{0} j=0}^{\mathrm{W}=1} P_{\mathrm{i}_{j} j}(i-j)^{2} \\
& \text { Energy }=\sum_{i_{j} j=0}^{N-1} P\left(i_{i} j\right)^{2} \\
& \text { Homogenity }=\sum_{\mathrm{i}_{0} j=0}^{N-1} \frac{P\left(\mathrm{i}_{0}\right)}{R} \\
& \text { Correlation }=\sum_{i_{i} j=0}^{N-1} P\left(i_{i} j\right)\left[\frac{R}{\sqrt{\left(i-\mu_{i}^{2}\right)\left(\sigma_{j}^{2}\right)}}\right]
\end{aligned}
$$

On the basis of above defined feature extraction approaches various features have been extracted from the images so that these features can be utilized for extraction of relevant images from dataset images.

These images have been retrieved based on distance classifier. Distance classifier computes distance between query image feature and dataset image features.

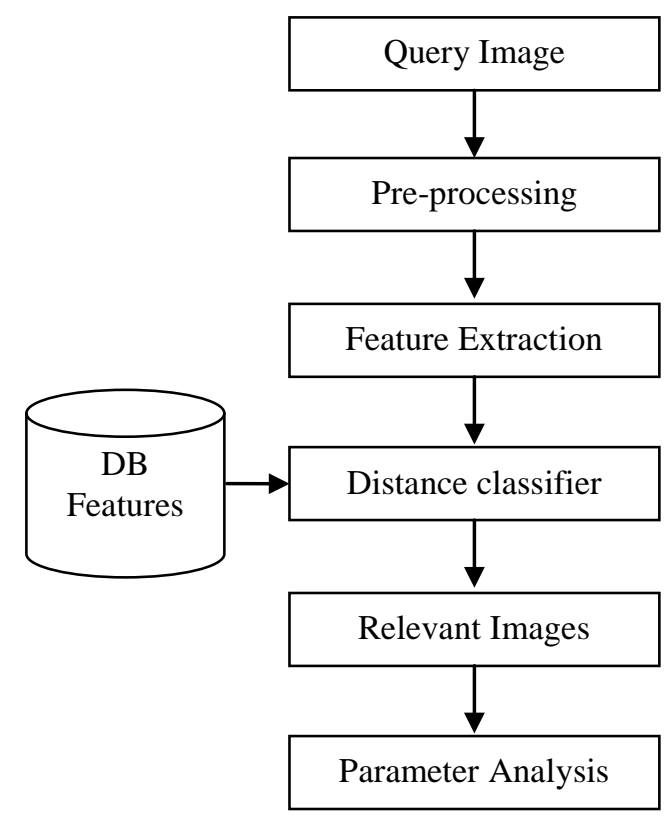

Fig 3.1 Flow of CBIR Model

Figure 3.1 represents step wise flow of the proposed system that has been used for extraction of relevant images from the dataset images. In this process numbers of relevant images have been retrieved from the dataset that are relevant to dataset images.

\section{RESULTS}

In the proposed work content based image retrieval has been done using shape color and texture features. On the basis of these features relevant images have been extorted from the dataset images. 
Corel 1-K dataset has been used in the proposed model that contains images of 10 different categories. These categories are African people, beach, buses, buildings, dinosaurs, elephants, horses, flower, mountains, and food. In the dataset each category contains 100 images. Feature for entire dataset has been computed and used for extraction of relevant images to the content of query image.

On the basis of extraction of relevant images different parameters have been analyzed for proposed system. These parameters are precision, recall and computation time.

- Precision: it is the fraction of the total relevant images to the total retrieved images that are extracted from the dataset.

$$
\text { Precision }=\frac{\text { Total relevent images }}{\text { Total images retrived }}
$$

- Recall: it is the fraction of the relevant image to the total no of relevant images available in the dataset.

$$
\text { Recall }=\frac{\text { Total relevent images }}{\text { Total relevant images in database }}
$$

- Computation Time: it is measure to check the time complexity of the algorithm. This computes total time taken by the algorithm for feature extraction.

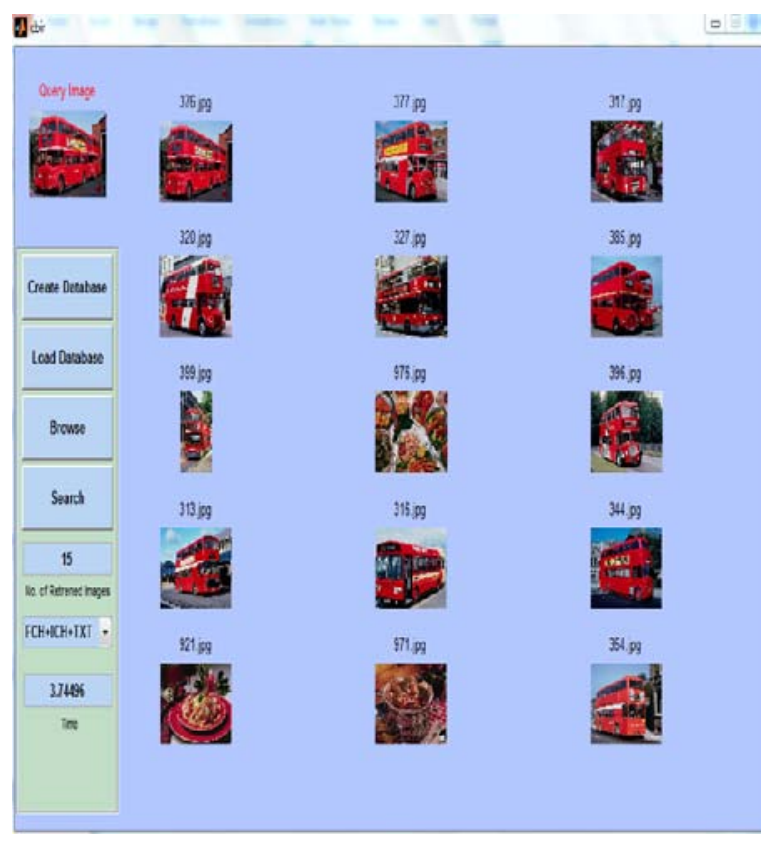

Fig 4.1 Retrieved images based on bus category

Figure 4.1 represents images that have been retrieved on the basis of bus image that has been given to the system as input image. On the basis of input image features of color compositions, texture and shape has been computed that provide information about relevant images.

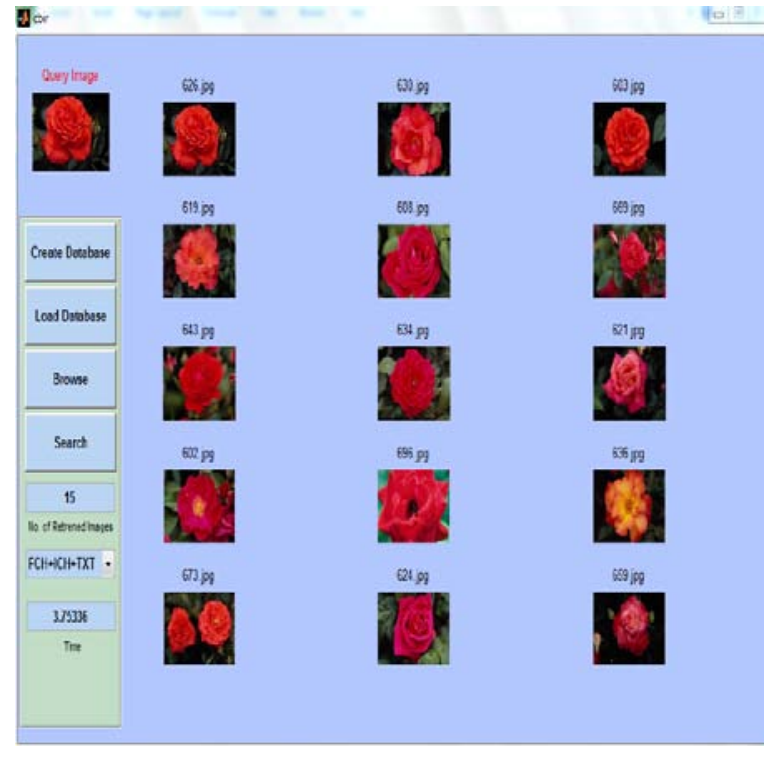

Fig 4.2 Retrieved images based on flower category

Figure 4.2 represents images that have been retrieved on the basis of flower image that has been given to the system as input image. On the basis of input image features of color compositions, texture and shape has been computed that provide information about relevant images.

In the proposed work 15 images has been retrieved based on query image. On the basis of these 15 images performance evaluation parameters have been evaluated for proposed system.

Table 4.1 Parameter Analysis of Proposed Approach

\begin{tabular}{|c|c|c|c|}
\hline $\begin{array}{c}\text { Database } \\
\text { category }\end{array}$ & Precision & Recall & $\begin{array}{c}\text { Computati } \\
\text { on time }\end{array}$ \\
\hline Africa & 0.8 & 0.12 & 5.27 \\
\hline Beach & 0.73 & 0.11 & 4.86 \\
\hline Building & 0.67 & 0.1 & 5.12 \\
\hline Bus & 1 & 0.15 & 4.95 \\
\hline Dinosaur & 0.93 & 0.14 & 4.96 \\
\hline Elephant & 0.67 & 0.1 & 5.35 \\
\hline Flower & 1 & 0.15 & 5.17 \\
\hline Food & 0.93 & 0.14 & 4.95 \\
\hline Horses & 0.87 & 0.13 & 4.78 \\
\hline Mountain & 0.6 & 0.09 & 4.91 \\
\hline Average & 0.82 & 0.12 & 5.03 \\
\hline
\end{tabular}

Table 4.1 represents performance evaluation parameters that have been computed for proposed work on different category basis and for over all image dataset on the basis of average values. 


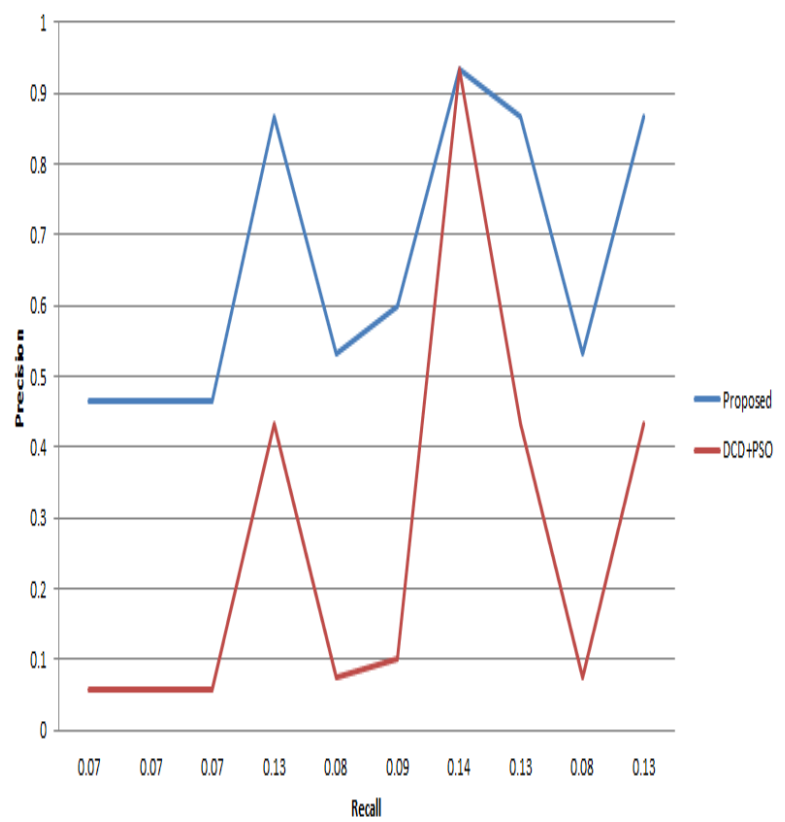

Fig 4.3 Comparative Analysis based on precision recall curve

Figure 4.3 represents comparison between proposed approach and existing approach in graphical representation. This graph represents precision and recall curve for both approaches. On the basis of these curve comparison can be made between proposed and existing approach.

\section{CONCLUSION}

Due to utilization of digital media information in the dataset contain large number of images. To retrieve similar images is a challenging task from large datasets. Various researches and development had been undertaken in the past years for development of effective content based image retrieval system. In proposed model an approach has been developed that is combination of color, shape and texture features for extraction of relevant images from the dataset. On the basis of these approaches features have been computed and used for extraction of relevant images. The proposed system provides $83 \%$ precision and low time complexity.

\section{REFRENCES}

[1] Hiwale, S.S.; Dhotre, D. "Content-based image retrieval: Concept and current practices” IEEE conference on Electrical, Electronics, Signals, Communication and Optimization (EESCO), 2015, pp. 1 - 6

[2] Dharani, T. Aroquiaraj, I.L., “A survey on content based image retrieval” IEEE conference on Pattern Recognition, Informatics and Mobile Engineering (PRIME), 2013, pp. 485 -490 .

[3] Murugan, M.V. Mathews, M.S. "2D and 3D active shape model with SURF algorithm for OBJECT retrieval: Content Based Image Retrieval” IEEE conference on Advanced Computing and Communication Systems (ICACCS), 2013, pp. 1-7.

[4] Pradeep, S.; Malliga, L. "Content based image retrieval and segmentation of medical image database with fuzzy values" IEEE conference on Information Communication and Embedded Systems (ICICES), 2014, pp. 1 - 7

[5] Bing Wang;XinZhang; Ziao-Yan Zhao; Zhi-De Zhang, “A semantic description for content-based image retrieval” IEEE conference on Machine Learning and Cybernetics, 2008, pp. 2466 - 2469.

[6] Kavitha, N. Jeyanthi, P. "Exemplary Content Based Image Retrieval using visual contents \& genetic approach”, IEEE conference on Communications and Signal Processing (ICCSP), 2015, pp.1378 - 1384

[7] Shiv Ram Dubey, Satish Kumar Singh, Rajat Kumar Singh,"Multichannel Decoded Local Binary Patterns for Content-Based Image Retrieval”, IEEE transactions on image processing, 2016,pp. 4018-4025

[8] C. R. Shyu, C. E. Brodley, A. C. Kak, “Assert: A physicianin-the-loop content-based retrieval system for hrct image databases," Computer Vision and Image Understanding, vol. 75, no. 12, pp. 111-132, 1999.

[9] T. M. Lehmann, T. Deselaers, H. Schubert, "IRMA - a content-based approach to image retrieval in medical applications,” In Information Resources Management Association, pp. 911-912, 2006

[10] W. Y. Ma, B. S. Manjunanth, "Netra: A toolbox for navigating large image databases," In Proceedings of International Conference on Image Processing, 1997.

[11] N. Boujemaa, J. Fauqueur, M. Ferecatu, "IKONA: Interactive generic andspecific image retrieval," In Proceedings of International workshop on Multimedia Content-Based Indexing and Retrieval(MMCBIR), 2001.

[12] J. Z. Wang, J. Li, G. Wiederhold, "SIMPLICITY: Semanticssensitive integrated matching for picture libraries," IEEE Transactions on Pattern Analysisand Machine Intelligence, vol. 23, no. 9, pp. 947-963, 2001. 